\title{
Espaces, parcours cérémoniels et fabrication d'objets rituels dans la fête mexica d'etzalcualiztli
}

Spaces, ceremonial paths and the manufacture of ritual objects in the Mexica festival of Etzalcualiztli

Espacios, recorridos ceremoniales y fabricación de objetos rituales en la fiesta mexica de Etzalcualiztli

Elena Mazzetto

\section{OpenEdition}

\section{Journals}

Édition électronique

URL : https://journals.openedition.org/jsa/13697

DOI : 10.4000/jsa. 13697

ISSN : 1957-7842

\section{Éditeur}

Société des américanistes

Édition imprimée

Date de publication : 17 septembre 2014

Pagination : 45-67

ISSN : 0037-9174

Référence électronique

Elena Mazzetto, «Espaces, parcours cérémoniels et fabrication d'objets rituels dans la fête mexica d'etzalcualiztli », Journal de la Société des américanistes [En ligne], 100-1 | 2014, mis en ligne le 01 janvier 2016, consulté le 04 septembre 2022. URL : http://journals.openedition.org/jsa/13697 ; DOI : https:// doi.org/10.4000/jsa. 13697 


\title{
ESPACES, PARCOURS CÉRÉMONIELS ET FABRICATION D'OBJETS RITUELS DANS LA FÊTE MEXICA D'ETZALCUALIZTLI
}

\author{
Elena MAZZETTO *
}

\begin{abstract}
Les descriptions des cérémonies religieuses des dix-huit fêtes de l'année solaire mexica que nous ont laissées les sources historiques de l'époque coloniale offrent une grande quantité de données sur la vie rituelle des Mexica. Néanmoins, la dimension spatiale relative à la caractérisation et à la distribution des lieux de culte, ainsi que les itinéraires urbains empruntés par les participants constituent des thèmes de recherche qui n'ont pas été suffisamment approfondis. Dans cette contribution, nous nous proposons de traiter ce sujet à propos de la fête d'etzalcualiztli, consacrée aux divinités aquatiques, en insistant sur trois aspects : l'utilisation de certains lieux religieux, l'élaboration d'objets rituels et la logique des déplacements cérémoniels réalisés dans l'espace urbain de Mexico-Tenochtitlan. [Mots-clés : rites, espaces sacrés, objets rituels, MexicoTenochtitlan, Mexica.]
\end{abstract}

Spaces, ceremonial paths and the manufacture of ritual objects in the Mexica festival of Etzalcualiztli. The colonial period historical descriptions of the eighteen celebrations of the Mexica solar year religious ceremonies offer a rich set of data on Mexica's ritual life. Nevertheless, the spatial dimension regarding the characterization and the distribution of the places of worship, as well as the urban itineraries taken by the participants are research topics that have not been investigated enough. In this work, we intend to approach these topics, in the case of the etzalcualiztli celebration dedicated to the aquatic gods, by drawing the attention on three aspects: the use of religious places, the creation of ritual objects and the logic of the ceremonial processions which took place in the urban space of Mexico-Tenochtitlan. [Key words: rituals, sacred spaces, ritual objects, Mexico-Tenochtitlan, Mexica.]

Espacios, recorridos ceremoniales y fabricación de objetos rituales en la fiesta mexica de Etzalcualiztli. Las descripciones de las ceremonias religiosas de las dieciocho fiestas del año solar mexica que nos dejaron las fuentes históricas de la época colonial presentan ricas series de datos acerca de la vida ritual de los mexicas. Sin embargo, la dimensión

* Université Libre de Bruxelles, département Histoire, Art et Archéologie, CP 175, 50 avenue F. D. Roosevelt, B-1050 Bruxelles, Belgique [elena.mazzetto@yahoo.it].

Journal de la Société des Américanistes, 2014, 100-1, pp. 45-67. (C) Société des Américanistes. 
espacial vinculada a la caracterización y a la distribución de los lugares de culto, así como los itinerarios urbanos seguidos por los participantes constituyen temas de investigación que no han sido suficientemente explorados. En este trabajo, nos proponemos abordar estos temas en el caso de la fiesta de etzalcualiztli, consagrada a las divinidades acuáticas, con especial enfoque en tres aspectos: la utilización de algunos lugares religiosos, la elaboración de objetos rituales y la lógica de los desplazamientos ceremoniales realizados en el espacio urbano de México-Tenochtitlan. [Palabras claves: ritos, espacios sagrados, objetos rituales, México-Tenochtitlan, mexicas.]

La vingtaine d'etzalcualiztli - « consommation d'etzalli », un repas de maïs et haricots - était consacrée aux Tlaloque ${ }^{1}$, les dieux de la pluie. En 1519, elle se célébrait entre le 24 mai et le 12 juin et le clergé faisait office de sacrificateur (Graulich 1999, p. 361). Les prêtres allaient recueillir des roseaux appelés aztapilli ou tolmimilli dans une source localisée près de la montagne Citlaltepec ${ }^{2}$, située au nord du bassin de Mexico, puis ils les liaient en paquets et les rapportaient dans la ville à l'aide d'un mecapalli ${ }^{3}$. Personne ne parcourait le chemin emprunté par les prêtres, car ceux-ci avaient le droit d'agresser et de voler les personnes qu'ils rencontraient ${ }^{4}$. Quand ils rejoignaient la ville, commençait alors la fabrication de sièges et de nattes faits avec les roseaux. Après quatre jours de pénitence, une grande procession rejoignait la lagune et les quatre ayauhcalli, les « maisons de brume », au bord de l'eau (Sahagún 1989, I, p. 125). Le début de la fête coïncidait avec la préparation et la consommation de l'etzalli par tous les habitants de Tenochtitlan. La danse de l'etzalli - à laquelle participaient des guerriers et des femmes de joie qui allaient d'une maison à l'autre portant des « lunettes » de feuilles sur leurs yeux - commençait à minuit. À l'aube, tous les prêtres partaient pour une autre procession, qui amenait les religieux punis pour avoir commis des fautes pendant les rituels, au Totecco, lieu de culte localisé dans l'aire septentrionale de Mexico-Tenochtitlan, à proximité du lac. Le jour de la fête, à minuit, avait lieu le sacrifice des captifs et, ensuite, celui des ixiptla, des dieux de la pluie, dans le Temple de Tlaloc au sommet du Templo Mayor. Les prêtres rejoignaient alors le Tetamazolco, le môle oriental de la capitale mexica, où ils embarquaient afin de rejoindre le Pantitlan, le tourbillon du lac Texcoco, où l'on jetait les offrandes ainsi que les cœurs des victimes sacrifiées (Sahagún 1950-1982, II, pp. 78-90).

À partir de ce résumé, l'intention principale de ce travail est d'explorer la liturgie spatiale de la vingtaine d'etzalcualiztli. Nous partirons de l'analyse de certains des espaces sacrés urbains impliqués et des itinéraires cérémoniels effectués, en proposant des localisations et l'agencement spatial de celles-ci. Notre démarche confirmera l'importance de la dimension insulaire de MexicoTenochtitlan, assez négligée jusqu'à présent. L'analyse des lieux et des parcours se combinera avec l'étude des végétaux employés pour la réalisation des étapes du culte - les nattes de roseaux aztapilin. Nous verrons que leur symbolisme fortement attaché à la pluie - renforce l'idée que l'espace, les mouvements et les 
objets rituels configurent un ensemble liturgique cohérent, conforme à la cosmologie indigène. À travers cet exemple, nous pensons ouvrir des pistes de recherche nouvelles, concernant, en premier lieu, la définition et les fonctions d'espaces déterminés - comme le calmecac - et l'utilisation de plusieurs lieux sacrés, ce qui constitue un reflet fidèle de la capacité de « fusion et fission » des divinités mexica.

L'étude des documents ayant trait à la vie religieuse des anciens Nahuas démontre que les informations relatives aux espaces où ils pratiquaient leur culte sont très nombreuses. Grâce à des ouvrages tels que l'Historia general de Sahagún (1989) ou l'Historia de las Indias de Durán (1984), on peut connaître les moments d'utilisation d'un espace et sa localisation au sein du tissu urbain de MexicoTenochtitlan ou encore l'identité des participants aux rituels qui s'y déroulaient. Plusieurs auteurs ont développé des analyses détaillées sur les rites réalisés tout au long de l'année solaire, sur les relations privilégiées avec les instances à l'honneur dans chaque rite et sur la dimension mythique évoquée dans la sphère cérémonielle ${ }^{5}$. Néanmoins, si l'on exclut la littérature inhérente au Templo Mayor et à l'enceinte sacrée, l'histoire de la recherche ne comporte que peu de publications portant, de manière approfondie, sur la dimension spatiale de la liturgie. Parmi les seules études visant à reconstruire l'« espace urbain de la fête »-finalité de notre thèse de doctorat (Mazzetto 2012) - se détachent les travaux de Carrasco (1991), Rovira Morgado (2010), Rodríguez Figueroa (2010) et González González (2011). Le paysage rituel du Bassin de Mexico, lui, a fait l'objet d'une série remarquable de publications de la part de Broda (1991a, 1991b, 1997, 2001) et Aveni (1991), les deux auteurs analysant à une plus grande échelle les relations entre l'espace naturel et la cosmogonie indigène. Notre point de départ théorique se fonde sur les résultats des recherches de López Austin (1983) à propos du dynamisme du panthéon mexica et de ses métamorphoses : l'union de plusieurs entités dans un seul être, porteur des attributs des différentes instances originales, ou, à l'inverse, la fragmentation d'une personnalité divine en divers aspects, avec une subdivision des caractéristiques la composant. Partant de ce phénomène double et antithétique, notre enquête s'est centrée sur l'utilisation des espaces consacrés au culte pendant les fêtes des vingtaines. Concernant ces espaces culturels, on a adopté les hypothèses formulées par Carrasco (1991) sur les caractéristiques des lieux sacrés des centres du pouvoir mexica et, notamment, sur les relations entre le centre et la périphérie. Dans le travail de López Austin - qui naît aussi en tant que réponse et approfondissement aux propositions de Smith (1992) à propos de la «vision locative de l'homme dans le cosmos »-, le rapport liant l'idée de « lieu » et celle de « performance » est la clé utilisée pour approcher la notion de « paysage cérémoniel » : un espace qui se constitue à travers une série de performances qui sont riches, en même temps, d'informations sur l'ordre social et symbolique mexica. Des travaux antérieurs, on retiendra aussi l'intérêt de la méthodologie éminemment pluridisciplinaire pratiquée par beaucoup et qui se fonde sur un dialogue entre sources écrites, pictographiques et 
archéologiques, sans exclure l'apport des données d'archives relatives à la première époque coloniale et à la localisation des anciens quartiers indigènes (Rovira Morgado 2010 ; González González 2011) ${ }^{6}$. Ce qui nous est en fait apparu manquer, ce sont des travaux monographiques sur les déplacements entre les différents lieux de manifestation du pouvoir surnaturel, exception faite de quelques analyses limitées et dispersées dans des publications de nature générale sur les rituels des anciens Nahuas (Dibble 1980; Broda 1991b ; Olivier 1997 ; Graulich 1999 ; González González 2011). Nous parlerons ici de «parcours cérémoniel » pour désigner les six catégories qui ont pu être isolées dans l'étude du Codex de Florence : les processions circulaires - tlayahualoliztli-, les déplacements des images divines, les batailles rituelles, les défis agonistiques, les déplacements des prêtres et, enfin, les mouvements qui saturaient l'espace.

\section{LES ESPACES DE CULTE À L'INTÉRIEUR DE L'ENCEINTE CÉRÉMONIELLE}

En raison de l'importance des ministres du culte lors de la vingtaine d'etzalcualiztli, les espaces formés par les demeures sacerdotales jouent un rôle majeur dans le déroulement des cérémonies. C'est précisément cet aspect qui permet d'approfondir l'une des fonctions de ces monastères, souvent négligée par les spécialistes, c'est-à-dire celle de lieux de fabrication d'objets rituels.

Les sources du $\mathrm{XVI}^{\mathrm{e}}$ siècle dépeignent les calmecac comme des institutions religieuses principalement vouées à l'éducation des jeunes gens appartenant à la classe dirigeante mexica, ainsi qu'aux pénitences sanglantes, apanage du clergé. C'est essentiellement dans cette optique qu'ils ont été étudiés tout au long de l'histoire (López Austin 1985; León-Portilla 1987; Calnek 1988). À notre connaissance, aucune publication n'a attiré l'attention sur le rôle majeur joué par les résidences sacerdotales dans la liturgie, en dehors de leur place comme lieux de préparation d'effigies divines comestibles, de vêtements portés par les représentations divines et de nourriture rituelle. Pourtant, les sources dévoilent d'autres détails fort intéressants. S'agissant d'activités qui étaient l'apanage des prêtres, on peut concevoir que l'espace concerné ait coïncidé avec le calmecac. Mais les informations contenues dans les documents coloniaux méritent d'être mieux analysées pour repenser les fonctions des monastères et en donner une image plus complète, allant au-delà des stéréotypes.

Le texte nahuatl du Codex de Florence ne fait aucune mention du lieu où les prêtres réalisaient les aztapilpetlatl (« nattes de roseaux »), tandis que sa version espagnole dit : " En llegando con las juncias al cu donde eran menester [...] ». Anderson et Dibble (Sahagún 1950-1982, II, p. 79) ainsi que Broda (1971, p. 282) suivent la version espagnole et traduisent le passage par : "And when they had come reaching [the temple]... » où « temple » est la traduction du mot cu. Or il s'agit d'un vocable que Sahagún utilise pour décrire des temples sur pyramide, des petites structures qualifiées aussi d'oratoires, de petits temples sans couverture, 
ainsi que des brasiers où on allumait le feu (Couvreur 2000, p. 72). Dans ce contexte, il faudrait traduire le mot $c u$ par teopan ${ }^{7}$, locatif formé sur teotl, « dieu » et le suffixe -pan, " près de », une référence probable à l'ensemble des édifices qui formaient l'enceinte à l'intérieur de laquelle se trouvait le sanctuaire du dieu ${ }^{8}$. Cet enclos comprenait, entre autres, le calmecac et le tzompantli. De plus, la formule " in teupan, in calmecac » est utilisée pour faire référence à la totalité des espaces contenus dans l'enclos d'un sanctuaire (Sahagún ibid., III, p. 51 ; VII, p. 30).

Notre interprétation, selon laquelle la fabrication des sièges et des nattes avait lieu dans un calmecac, est renforcée par l'analyse des autres vingtaines. En atemoztli, c'étaient les prêtres des divinités de la pluie qui étaient chargés d'habiller et orner les Tepictoton, les petites effigies divines des dieux des montagnes, dans le calmecac, et non dans les maisons (ibid., II, p. 152). Une fois le sacrifice des Tepictoton terminé, c'était au calmecac que les gens apportaient la pâte d'amarante tzoalli, c'est-à-dire « le corps des effigies-montagnes » (ibid., I, p. 48). En xocotl huetzi, les vieux prêtres (cuacuacuiltin) avaient la charge de façonner le Xocotl - la figure de pâte d'amarante que l'on mettait au sommet du mât - et de l'orner (ibid., II, p. 112). En panquetzaliztli, on cuisinait la pâte utilisée pour la fabrication de l'effigie de Huitzilopochtli dans un lieu appelé Tilocan ou Xilocan (López Austin 1965, p. 99), qualifié de « maison ». Ensuite, les prêtres façonnaient l'effigie dans la "maison » appelée Itepeyoc. Dans sa Monarquía Indiana, Torquemada (1975-1983, VI, p. 113) explique que ce rituel avait lieu dans une des salles du temple, "que era cerca de su altar y cu ». Le croisement de ces données avec celles contenues dans la Historia de las Indias de Durán (1984, I, pp. 27-28) permet d'affirmer que le Xilocan pourrait être identifié comme étant un calmecac féminin rattaché au Grand Temple. Les fonctions principales des jeunes femmes étaient de préparer à manger pour la divinité ainsi que de réaliser et broder les vêtements portés par les effigies, ce qui confirme qu'une des fonctions des résidences des prêtres était la réalisation d'objets rituels (Sahagún 1950-1982, XII, p. 51 ; Durán ibid., pp. 26-27). En toxcatl, l'élaboration d'une effigie en pâte de Huitzilopochtli avait lieu dans le temple de Huitznahua et, par extension, dans le quartier du dieu (Sahagún ibid., II, p. 71) tandis que la représentation élaborée dans le quartier de Huitznahuac était celle du compagnon de Huitzilopochtli, Tlacahuepan Cuexcotzin (ibid., p. 192). Il y avait donc des espaces spécifiques consacrés aux différentes étapes de création des effigies divines, qui faisaient probablement partie du calmecac et, dans le cas des quartiers, des oratoires calpulco ${ }^{9}$. Il n'est donc pas hasardeux de formuler la même hypothèse concernant l'élaboration d'objets rituels.

Dans son Annexe, Sahagún (1950-1982) mentionne la présence de sept demeures sacerdotales ${ }^{10}$. Même si, en etzalcualiztli, on fait référence de manière générale aux calmecac présents dans la ville, on peut émettre une hypothèse par rapport à la localisation de la résidence sacerdotale d'où sortait la procession des prêtres qui se dirigeait au Temple de Tlaloc, avant le sacrifice des représentations 
divines. Cette procession était guidée par le Tlalocan tlenamacac, le rang le plus élevé du clergé mexica, et il est donc légitime de croire que le calmecac dont il est question dans ce passage est le Mexico Calmecac, situé dans l'enceinte sacrée (Sahagún ibid., p. 182 ; 1989, I, p. 183). En effet, en etzalcualiztli, le Tlalocan tlenamacac fait son apparition seulement au moment de la procession qui mène le clergé du calmecac au Templo Mayor (Sahagún 1950-1982, II, p. 87). Il n'est jamais question de son intervention dans les espaces situés en dehors du centre cérémoniel. Marquina (1960, pp. 100-102), en suivant le schéma de l'illustration de l'enceinte sacrée contenue dans les Primeros Memoriales (Sahagún 1997), avançait que le calmecac se situait dans la partie nord-ouest de l'enceinte, avec une orientation nord-sud. Des fouilles récentes du Programa de Arqueología Urbana ont permis la découverte et le dégagement partiel de ce qui semble être ce calmecac, sous le Centre culturel espagnol, Calle Donceles $\mathrm{n}^{\circ}$ 97. La couche archéologique d'époque préhispanique comprenait trois étapes de construction et la chronologie de l'édifice correspond à l'étape VI du Templo Mayor, période qui s'étend de 1486 à 1502 apr. J.-C. (Barrera Rodríguez et López Arenas 2008, pp. 22-29). La demeure des prêtres devait donc être située à l'extrémité nordoccidentale de l'enceinte sacrée et la procession décrite en etzalcualiztli aurait donc suivi un parcours ouest-est, un mouvement réitéré - comme on le verra dans la plupart des autres parcours de la fête.

\section{LES ESPACES DE CULTE EN DEHORS DE L'ENCEINTE CÉRÉMONIELLE}

\section{Le Totecco}

Ce lieu de culte - dont le nom a été traduit par « dans notre seigneur » par Garibay (González González 2011, p. 122) ou par « le lieu de notre serpent » par Arnold (2009, p. 189) - était visité lors de la punition infligée aux ministres du culte. Les prisonniers étaient jetés dans tous les points d'eau que les religieux rencontraient sur leur chemin, puis les prêtres brûlaient des papiers sacrificiels et des figurines de dieux faites d'encens et de caoutchouc. Ils étalaient ensuite l'herbe yauhtli sur les nattes de roseaux. Les prêtres capturés étaient jetés dans l'eau et presque noyés (Sahagún 1950-1982, II, p. 86). Cette cérémonie avait un poids important dans la liturgie, puisqu'il s'agissait du point de rencontre de plusieurs prêtres du feu et que les familles des religieux transgresseurs s'y rendaient également. C'était ici, en tlacaxipehualiztli, que se terminaient les escarmouches entre les xipeme, c'est-à-dire les pénitents qui portaient une peau d'écorché, comme le dieu Xipe Totec, et les guerriers, et que se trouvait une statue en pierre de cette divinité (Sahagún ibid., IX, p. 70 ; González González ibid.). Dans ce passage du texte de Sahagún, cet espace est dénommé Totectzontecontitlan, « près des crânes de notre seigneur ». L'auteur spécifie aussi que le Totecco se trouvait là où avait été érigée l'église de la Conception, qui faisait partie du 
quartier tlatelolca d'Amaxac, et qu'il était caractérisé par la présence d'un embarcadère (ibid., XII, pp. 109-110). Caso (1956, p. 36) identifie ce quartier comme celui d'Atenantitlan, localisé à l'extrémité nord-orientale de l'île ${ }^{11}$. Les études de González Aparicio (1973, pp. 45-46) et González González (2011, p. 125) semblent confirmer que le Totecco était localisé près d'un chemin qui menait à Tepetzinco, la petite montagne au milieu de la lagune, où se situaient au moins deux ayauhcalli ${ }^{12}$ (Figure 1). Ce détail confirme l'étroite relation qui existait entre les principaux centres de pouvoir des puissances surnaturelles liées à l'eau, tout comme la vocation aquatique et nourricière de cet espace, lié au culte des dieux de la pluie et du maïs, sous les traits des Tlaloque et de Xipe Totec ${ }^{13}$. Or le lien qui existe entre les différents champs d'actions des divinités mexica est une caractéristique profondément structurelle de la pensée religieuse des anciens Mexicains, encore extrêmement vivante au sein des sociétés indigènes contemporaines. En ce qui concerne les dieux de la pluie et du maïs, nous savons qu'il s'agit d'un phénomène bien connu et qui remonte à une époque très antérieure au Postclassique ${ }^{14}$. À l'époque mexica, le cas sans doute le plus connu est celui du sanctuaire de Tlaloc, appartenant à la Phase II du Templo Mayor, à l'intérieur duquel se trouvent deux peintures murales de Cinteotl, le dieu du maïs mûr. Le culte pratiqué dans cet espace sacré périphérique suivait donc la même logique.

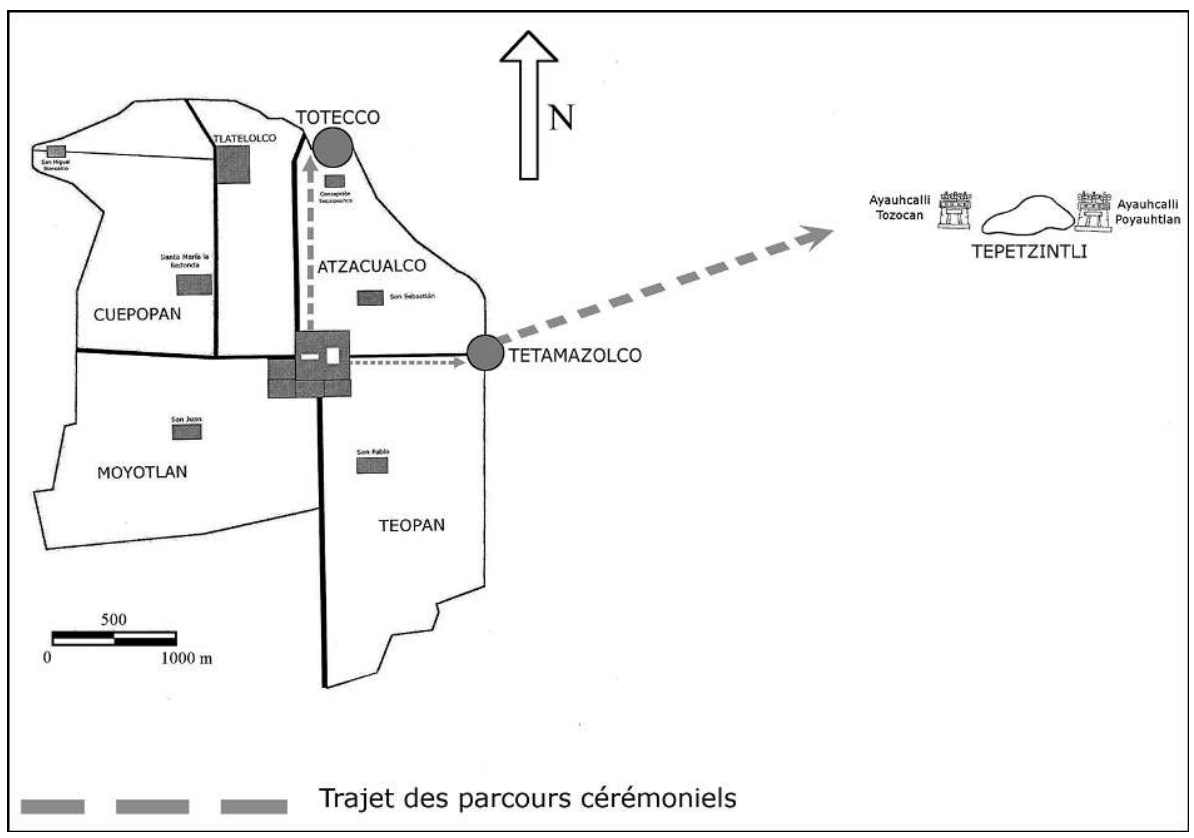

FIG. 1 - La localisation des espaces sacrés de la vingtaine d'etzalcualiztli à Mexico-Tenochtitlan. Dessin de l'auteur (E. M.) d'après González Aparicio (1973). 


\section{Le Tetamazolco}

Tetamazolco signifie « dans le crapaud de pierre ». Il s'agissait de l'embarcadère destiné aux bateaux localisés à la fin de la chaussée orientale de l'île de Mexico-Tenochtitlan, qui sortait du centre cérémoniel et qui correspond aux actuelles rues de República de Guatemala et Miguel Negrete (Lombardo de Ruíz 1973, pp. 137-138). C'est précisément à cet endroit que, durant huey tecuilhuitl, la représentation divine de la déesse du maïs Xilonen "entrait dans le sable » (Sahagún 1950-1982, II, pp. 103-104 ; Dibble 1980, pp. 197-202 ; López Austin et López Luján 2009, pp. 304-309) ${ }^{15}$. Pendant ce rituel, on réalisait une procession circulaire autour de la pyramide ou de la pierre du sacrifice située au sommet. Il est donc possible que le Tetamazolco ait également été caractérisé par la présence d'un temple ou d'un autel et d'un point d'eau où les ministres du culte allaient se baigner. Pendant huey tecuilhuitl, ce lieu était lié au signe acatl, le signe de l'est, un des quatre porteurs de l'année mésoaméricaine, et, par conséquent, à une véritable représentation spatiale du temps (Sahagún ibid., II, p. $104 ; 1989$, I, p. 138). Lombardo de Ruíz (1973, pp. 137-138) l'identifie à l'aire de l'actuelle église de San Lázaro. Néanmoins, ce lieu de culte n'était pas superposé au Tetamazolco d'époque mexica. En effet, les documents du Xvi ${ }^{e}$ siècle nous apprennent que, avant d'être érigé dans l'aire orientale de la ville, en 1572, le sanctuaire de San Lázaro avait connu un autre emplacement : il avait été construit entre 1521 et 1524, puis détruit et déplacé en 1528 (Sánchez Uriarte 2010, pp. 82-83). La cérémonie au Pantitlan se déroulait entre minuit et l'aube et se terminait avec le retour des prêtres à Tetamazolco, où l'on se baignait rituellement et où l'on punissait à nouveau les prêtres coupables de ne pas avoir respecté le jeûne pour Tlaloc. Nous attirons l'attention non seulement sur l'importance liturgique du lieu par rapport aux entités aquatiques, mais aussi sur les points qu'il avait en commun avec le Totecco. Tout comme le premier, il s'agissait d'un endroit riverain, où l'on punissait le clergé négligeant. Il était muni d'un embarcadère et permettait de rejoindre - par voie terrestre ou aquatique - le Tepetzintli et le Pantitlan. En outre, la liturgie du Tetamazolco convoquait une fois encore dans le même espace la liturgie aquatique des Tlaloque et celle, végétale, du maïs (Figure 1).

\section{LES PARCOURS CÉRÉMONIELS}

Les parcours effectués par les ministres du culte durant etzalcualiztli, constituent un bon exemple des différents déplacements qui avaient lieu durant les fêtes. Le cycle des vingtaines permet de souligner que ces derniers étaient toujours accompagnés de musique et de chants. Pour cette raison, les formes verbales qui 
les définissaient, pendant cette vingtaine, sont les suivantes : tlapitzalotiuh: « aller en faisant résonner les conques » et qujpitztiuh : «aller en soufflant sur quelque chose ». Ces termes sont utilisés pour décrire les déplacements - souvent nocturnes - effectués pour rejoindre les ayauhcalli, les sommets des montagnes ou les sanctuaires dédiés aux maîtres des pluies, autrement dit, pour rejoindre les centres du pouvoir des entités aquatiques. Olivier (1997, pp. 244-246) a souligné l'étroite relation qui existe entre le son des instruments à vent et la réalisation d'événements rituels comme la pénitence, la guerre, la chasse et le sacrifice. De même, il a soutenu que l'objectif probable de cette musique était d'attirer l'attention des instances surnaturelles afin de les inviter à descendre sur terre. Il s'agissait donc d'un puissant moyen de communication. En conséquence, il est logique d'imaginer un monopole des ministres du culte sur ces catégories d'instruments, moyen privilégié pour convoquer les êtres divins. Les réalisations de pénitences et les sacrifices n'étaient pas les seules occasions au cours desquelles on jouait de ces instruments. De fait, les parcours réalisés pour rejoindre les ayauhcalli avaient aussi pour but le dépôt d'objets rituels usagés. Par ailleurs, la manifestation de la réciprocité qui existe entre les instances divines et les hommes est bien exprimée par les mouvements spatiaux effectués. Les expéditions dans les montagnes avaient pour but d'aller chercher les instruments de pénitence ou de conduire des victimes vers les lieux de sacrifice. Les prêtres y recueillaient des outils mis à leur disposition par les divinités aquatiques pour l'autosacrifice; à l'inverse, les victimes offertes permettaient aux hommes de "payer leur dette » envers les dieux.

L'étude des parcours met en évidence un modèle radiant, dont les directions les plus utilisées sont le nord (le Citlaltepec, le Totecco) et l'est (le Tetamazolco, le Pantitlan), orient cosmique où étaient localisés la plupart des centres de pouvoir où se manifestaient les entités sacrées de l'eau et où se situait le Mont Tlaloc. Or la partie du monde correspondant à l'est, direction du lever du soleil, était appelée tlalocan. On note également que le vent provenant de l'est était appelé tlalocayotl, ce qui confirme la relation entre ce point cardinal et le dieu de la pluie (López Austin 1997, pp. 216-218). Le nord, pour sa part, s'appelait mictlampa, le nom également du vent provenant du pays des morts (Sahagún ibid., VII, p. 14). Broda (1991a, p. 113) le considère comme la direction cosmique d'où provenaient les pluies ${ }^{16}$.

\section{LE SYMBOLISME DES ROSEAUX AZTAPILIN ET LA DIMENSION VÉGÉTALE DE LA VINGTAINE}

Les roseaux, centre de la liturgie de la vingtaine d'etzalcualiztli, appelés aztapilin/oztopilin (ou tolmimilli ${ }^{17}$ ) étaient longs, hauts et avaient des bases très blanches (Sahagún ibid., XI, p. 195). Ils avaient pour dieu tutélaire 
Nappatecuhtli, «Le Quatre Fois Seigneur », un des dieux de la pluie (López Austin 1997, p. 119), grâce auquel ils poussaient (Sahagún ibid., I, p. 45). Pendant l'élaboration des sièges et des nattes, les prêtres juxtaposaient la partie blanche des bases et le vert des cannes. Les éléments confectionnés devenaient des instruments utilisés pendant le culte, avec lesquels les prêtres réalisaient les différentes étapes du rituel. Les nattes constituaient le lit des prêtres tout au long des vingt jours ; elles étaient aussi le siège où l'on déposait différentes offrandes de nourriture, de l'herbe yauhtli ainsi que des boules de jade, utilisées probablement pour évoquer les pluies, au pied du Templo Mayor (Graulich 1999, pp. 365-366 ; Dehouve 2012, p. 48). À première vue, la description du façonnage des nattes peut passer pour un texte fortement rhétorique, mais on doit remarquer que, dans le cycle des vingtaines, il n'y a aucune autre description aussi minutieuse de la fabrication d'objets rituels. En outre, les détails fournis nous permettent de reconnaître la présence des sièges tressés dans un bon nombre de documents pictographiques. L'étude du Codex de Florence met en évidence le caractère extraordinaire de l'utilisation des nattes tressées, qui apparaissent dans des contextes rituels et souligne la relation exclusive que ces végétaux entretenaient avec le culte aquatique et terrestre. Il ne faut pas croire d'ailleurs que toutes les plantes aquatiques étaient liées aux dieux de la pluie. Lors de la vingtaine, en effet, on avait recours à d'autres types de roseaux lacustres, pour des usages différents. Ainsi, les prêtres allaient d'abord chercher des branches de pin ou des roseaux verts, qui étaient apportés dans les temples des dieux. Ces roseaux, appelés acaxoxouhqui, faisaient partie des instruments de pénitence habituellement utilisés par les prêtres. Le mythe de la naissance du cinquième Soleil présente ces végétaux comme le véritable "prototype» des plantes employées par Nanahuatzin au moment du jeûne. Tecciztecatl-Lune avait utilisé, en revanche, des plumes de quetzal (Sahagún ibid., VII, p. 4 ; Graulich 2000, pp. 118-130). Plus tard et à un autre moment durant la fête, on rentrait au calmecac et on éparpillait par terre des roseaux appelés atolin ${ }^{18}$. Rappelons aussi que l'oztopilcuahuitl, le bâton de roseau oztopili, était un attribut distinctif des dieux de la pluie et des montagnes. Les petites effigies l'arboraient pendant atemoztli ou au moment où elles étaient placées devant les défunts qui allaient être enterrés dans les Maisons de Brume ${ }^{19}$. D'un point de vue iconographique, ces roseaux sont représentés dans plusieurs images du Codex Borbonicus (1991, pl. 23, 29, 32, 35) et du Codex Magliabechiano (1970, pl. 34r, 63r, 81r, 82r). Dans le premier document, ils jouent le rôle de siège, d'ornement de temples et de bouclier et sont associés à des divinités comme Tlaloc, Chicomecoatl, déesse du maïs mûr, et Nappatecuhtli (Figures 2, 3, 4). 


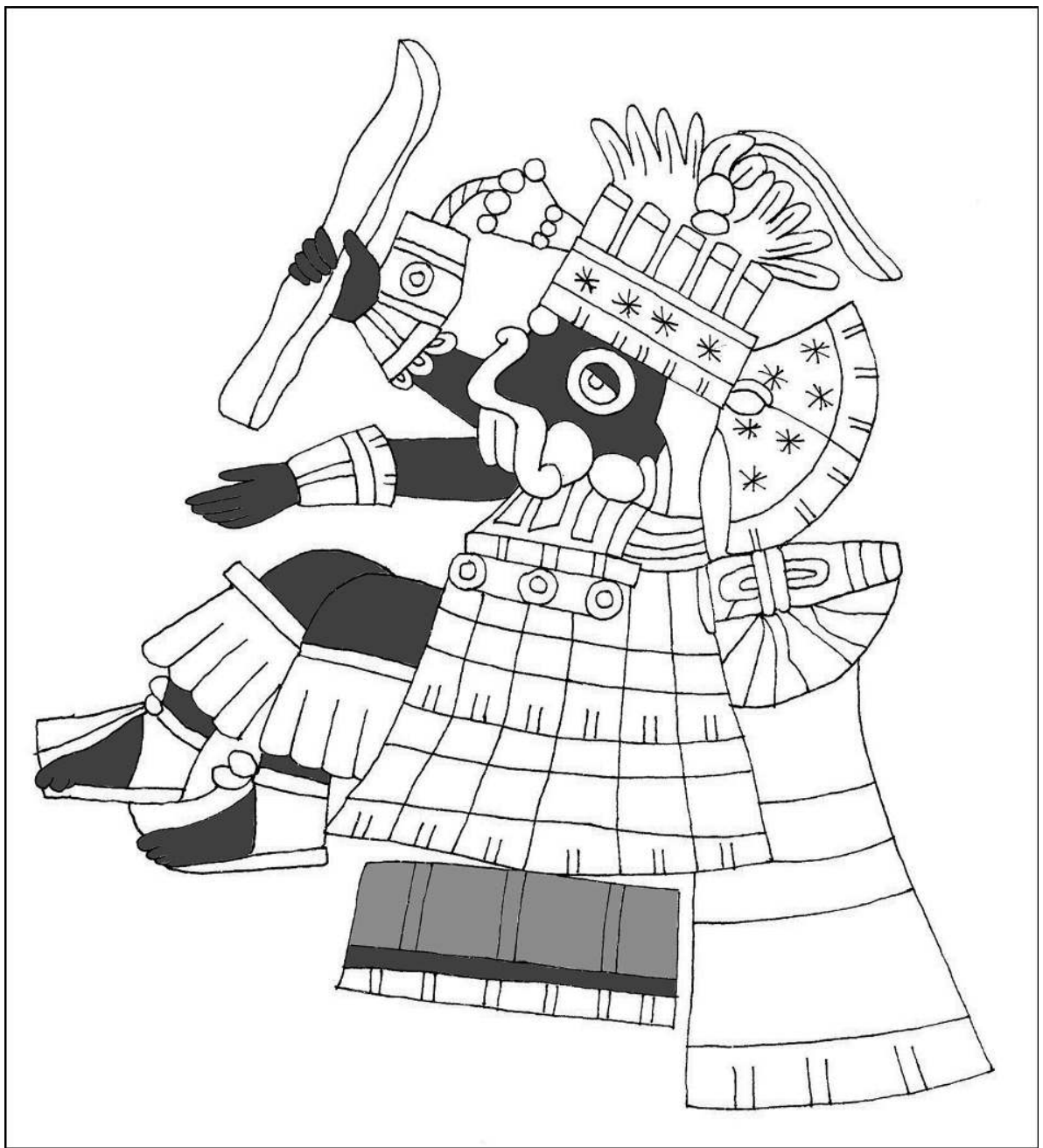

FIG. 2 - Tlaloc assis sur un siège de roseaux aztapilin. Dessin de l'auteur (E. M.) d'après le Codex Borbonicus (1991, pl. 23).

Dans le Codex Magliabechiano, en revanche, les dieux associés sont Tlaloc, Ixtlilton, entité liée au pulque, et une autre divinité interprétée comme l'un des démons nocturnes (Tzitzimime), ou bien la déesse de la terre Tlaltecuhtli, ou encore le dieu du pays des morts, Mictlantecuhtli (Figures 5, 6, 7). 


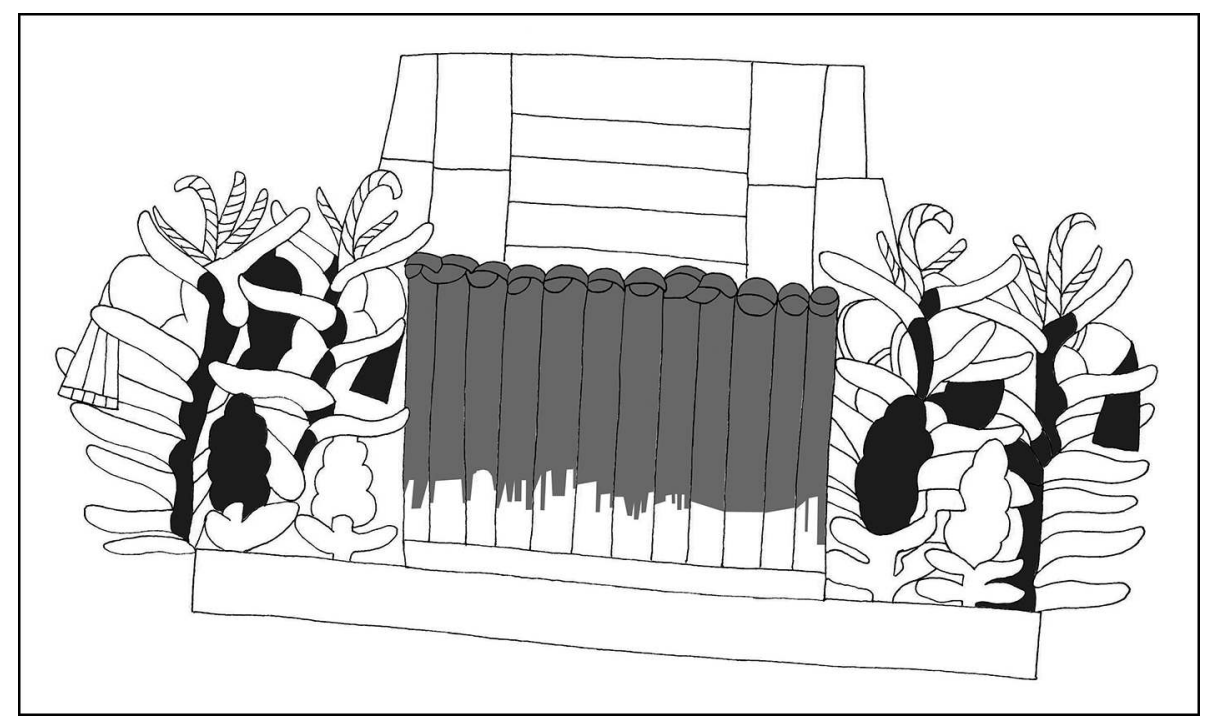

Fig. 3 - Le temple associé à Chicomecoatl, orné de roseaux aztapilin. Dessin de l'auteur (E. M.) d'après le Codex Borbonicus (1991, pl. 29).

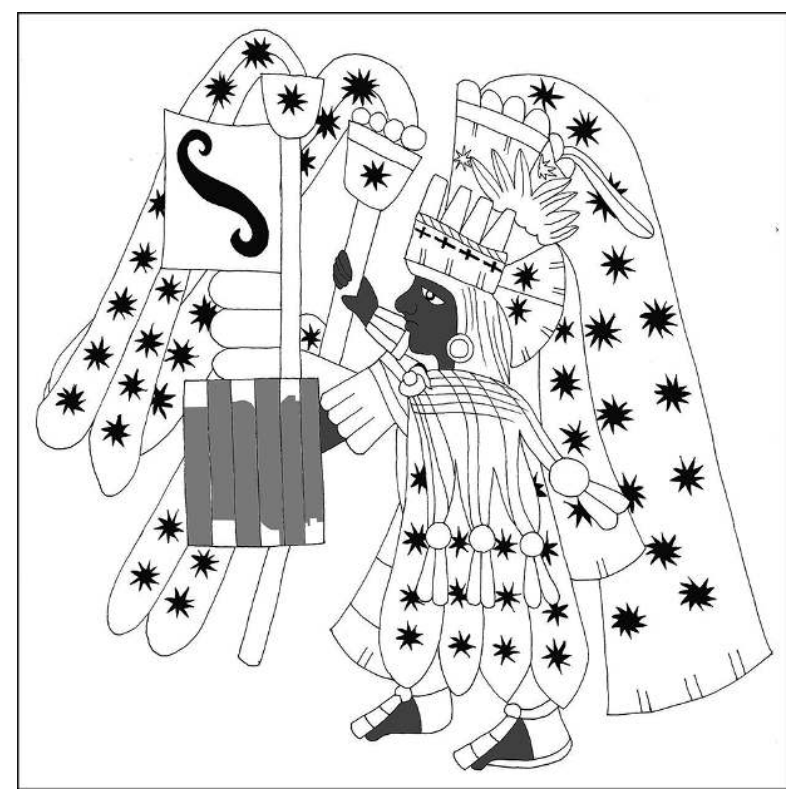

Fig. 4 - Nappatecuhtli avec un bouclier réalisé de roseaux aztapilin. Dessin de l'auteur (E. M.) d'après le Codex Borbonicus (1991, pl. 32). 


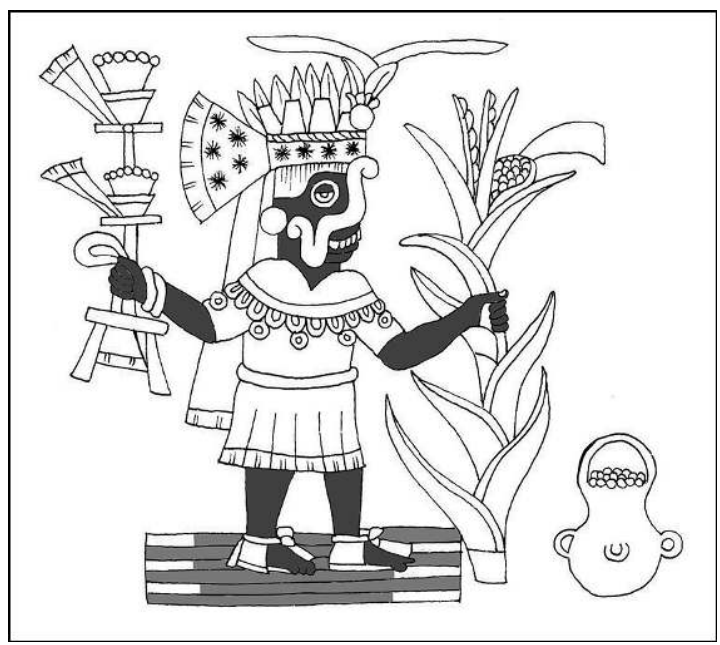

FIG. 5 - Tlaloc debout sur une natte aztapilpetlatl. Dessin de l'auteur (E. M.) d'après le Codex Magliabechiano (1970, pl. 34r).

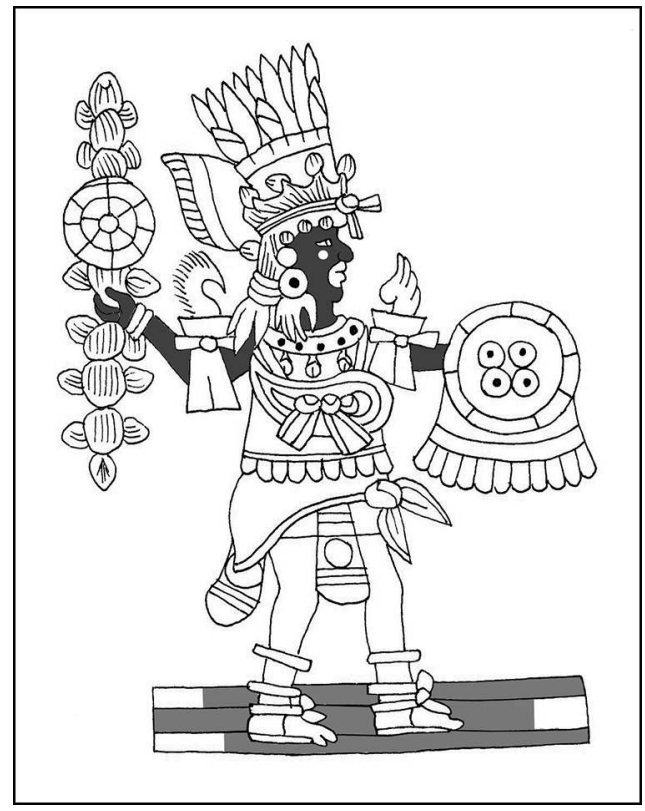

FIG. 6 - Ixtlilton debout sur une natte aztapilpetlatl. Dessin de l'auteur (E. M.) d'après le Codex Magliabechiano (1970, pl. 63r). 


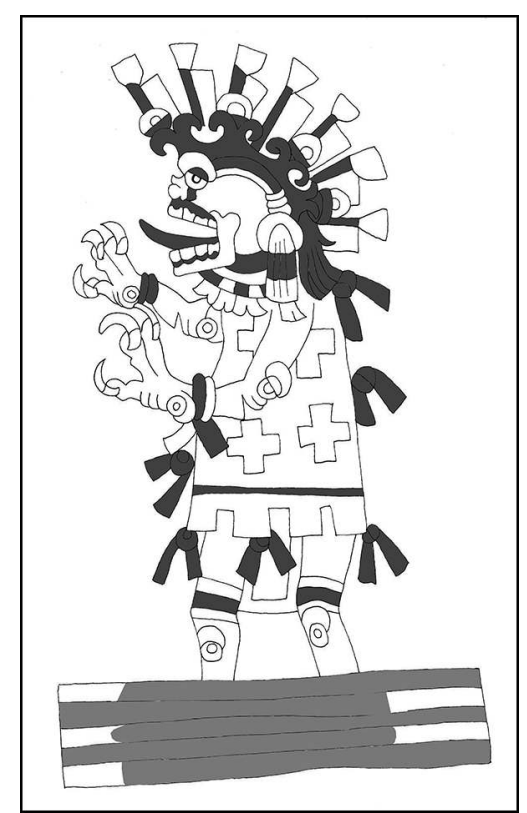

FIG. 7 - Tlaltecuhtli ou une Tzitzimitl debout sur une natte aztapilpetlatl. Dessin de l'auteur (E. M.) d'après le Codex Magliabechiano (1970, pl. 82r).

Durant les mois et fêtes tepeilhuitl et atemoztli, notre roseau est cité parmi les différents types de roseaux utilisés pour confectionner des lits de joncs où étaient dressés les dieux des montagnes (Sahagún ibid., II, pp. 132, 153). Or les divinités de la pluie partageaient plusieurs traits avec celles des montagnes et du vent. Elles s'apparentaient aussi à l'ensemble des dieux du maïs et, en général, à la terre, tout comme à ses auxiliaires, les Centzon Totochtin, dieux du pulque et de l'ivresse, autres incarnations des dieux des montagnes (López Austin 1997, p. 225). Voilà pourquoi nous retrouvons, parmi les effigies-Tepictoton - et parmi les utilisateurs des nattes - les noms de plusieurs Tlaloque, les dieux-montagnes qui sont aussi ceux du pulque. Dans ce dernier groupe, nous pouvons compter Ixtlilton ainsi que Chicomecoatl, qui s'avère être un Tlaloque (Sahagún ibid., VI, p. 35). Si, parmi les dieux des montagnes, les sources citent également Cihuacoatl et le dieu $\mathrm{du}$ feu, c'est en raison du caractère tellurique de Cihuacoatl-Ilamatecuhtli, l'épouse de Mictlantecuhtli, fêtée précisément pendant etzalcualiztli (Codex Ixtlilxochitl 1976, p. 21). Celle-ci se confond avec la déesse du foyer domestique, Chantico, les démons Tzitzimime et la déesse Tlaltecuhtli (Leyenda de los Soles, p. 191 ; Graulich 2000, p. 228). Son temple, le Tlillan, situé dans l'enceinte sacrée de Tenochtitlan, hébergeait aussi d'autres effigies divines dont la description fait fortement penser aux maîtres de la foudre (Durán 1984, I, p. 127 ; Broda 
1991b, p. 488). Quant au dieu du feu Huehueteotl-Xiuhtecuhtli, il était celui « qui est au centre de la terre » (Sahagún ibid., VI, p. 19). En tant que foyer domestique, il représentait le centre symbolique de chaque maison et possédait une dimension à la fois céleste, terrestre et infraterrestre (Ragot 2000, p. 117). Les aztapilpetlatl étaient donc des objets rituels façonnés pour recevoir des entités surnaturelles spécifiques, appartenant au domaine de l'eau et de la terre (López Austin 1997, pp. 199-200). Ces mêmes objets étaient recouverts d'herbe yauhtli et servaient de siège aux prêtres de Tlaloc, de supports aux boules de jade ainsi qu'aux offrandes alimentaires constituées par les boules de maïs, des tomates et des piments verts. Il s'agissait des différentes représentations - humaines, végétales ou minérales du domaine naturel de l'eau. Si la relation symbolique entre les dieux de la pluie, les prêtres de Tlaloc, les boules de jade et l'herbe yauhtli est un aspect bien connu dans le rituel mexica (Sahagún ibid., III, p. 47, VI, p. 35 ; Ortiz de Montellano 1980, pp. 290-294 ; Graulich 2005, p. 233), les offrandes alimentaires peuvent être considérées comme une manifestation du tonacayotl, la nourriture des hommes, dont Chicomecoatl était l'incarnation (Sahagún ibid., I, p. 13 ; Durán 1984, I, p. 135), ainsi qu'une manifestation des richesses aquatiques et terrestres du Tlalocan, le paradis du dieu de la pluie ${ }^{20}$. Il s'agit d'un véritable langage végétal, la liturgie prévoyant l'utilisation de plantes et de fleurs spécifiques pour chaque membre du panthéon. En effet, l'utilisation de végétaux dont le symbolisme pouvait être rattaché à une personnalité divine n'était pas une coutume propre à la fête d'etzalcualiztli; au contraire, le cycle des vingtaines nous en offre plusieurs exemples $^{21}$. Le cas le plus connu est celui de la vingtaine de tlacaxipehualiztli, pendant laquelle on fabriquait les tzapoicpalli - les sièges de sapotillier - utilisés par les prêtres sacrificateurs (Durán 1984, II, p. 173), ainsi que par les xipeme. Les grappes de maïs que les habitants de la ville accrochaient aux toits des maisons étaient également disposées sur ces sièges (ibid., I, p. 97 ; Sahagún ibid., I, p. 40 ; González González 2011, p. 274). Comme l'a signalé González González (ibid.), cette caractéristique démontre une équivalence substantielle entre les représentations divines du dieu et la céréale, au moment où elle avait atteint son stade définitif de maturation ${ }^{22}$. D'autres exemples - s'ils sont moins clairs - existent pour les vingtaines de huey tozoztli, toxcatl et quecholli. Pendant la fête des dieux du maïs, on jeûnait pendant quatre jours et de jeunes garçons ornaient les effigies des dieux avec des roseaux tolpatlactli, en coupant leurs parties inférieures. Ensuite, lors des jours de pénitence, les jeunes gens couvraient les effigies de sang issu de leurs oreilles et de leurs jambes (Sahagún ibid., II, p. 61 ; 1989, I, p. 113) ${ }^{23}$. En toxcatl, la cour du temple et les escaliers de la pyramide de Tezcatlipoca étaient parsemés de feuilles de maguey. Il existe une relation évidente entre cette plante, que nous savons liée à la production du pulque, et le culte de Tlamatzincatl, dieu de l'ivresse qui n'était autre qu'un aspect de Tezcatlipoca. Rappelons que les informateurs de Sahagún déclarent qu'il s'agissait de la fête de Tezcatlipoca " [...] y por otro [nombre] Tlamatzincatl » 
(Sahagún 1989, I, p. 115 ; voir Olivier 1997, p. 254) ${ }^{24}$. Finalement, dans certains des espaces sacrés de la vingtaine de quecholli - tels que la cour du temple de Mixcoatl - on étalait de l'herbe sèche zacate, un végétal dont le symbolisme se rattache au nord poussiéreux et aride, théâtre des aventures mythiques de Mixcoatl (Leyenda de los Soles, pp. 185-189 ; Sahagún 1950-1982, II, p. 226).

\section{Conclusions}

Dans cette étude, nous avons tenté de mettre en valeur certains aspects de la liturgie d'etzalcualiztli à travers l'analyse de trois espaces de culte, des itinéraires cérémoniels et des objets rituels utilisés, en attirant l'attention sur les nombreuses pistes de recherche que la démarche méthodologique que nous avons suivie révèle. Avant tout, nous avons vu que, en dépit de l'image officielle que les documents anciens leur ont attribuée, et qui a été reprise dans les recherches des spécialistes, les calmecac étaient des espaces multifonctionnels. La préparation d'objets rituels et de nourriture divine était une activité fondamentale de la liturgie et elle avait lieu dans les demeures sacerdotales. L'importance de ce point mérite un approfondissement étant entendu que le calmecac demeurait une institution dont la tâche principale était éducative et pénitentielle.

Ensuite, l'attention portée à Totecco et à Tetamazolco nous a permis d'approcher deux aspects majeurs de la dimension spatiale des liturgies de Mexico-Tenochtitlan. On a vu que ces lieux ont en commun de nombreuses caractéristiques : situés à la frontière entre la terre et les espaces aquatiques des lacs, il s'agissait d'endroits riverains qui faisaient office d'embarcadères, où avaient lieu des bains rituels ainsi que les punitions des prêtres coupables. Il s'agissait également de deux étapes obligatoires pour pouvoir rejoindre - par un chemin terrestre ou aquatique - le Tepetzintli et le Pantitlan. Leur localisation exacte dans le territoire insulaire nous permet de voir représentés les deux orients cosmiques impliqués dans le cycle saisonnier des pluies : l'est et le nord. Un tour d'horizon rapide des vingtaines montre d'ailleurs que ces deux espaces jouaient également un rôle significatif dans la liturgie des divinités se rapportant au maïs, comme Xipe Totec et Xilonen. L'étude des espaces religieux, lorsqu'une approche polysémique est privilégiée, met en évidence l'emploi régulier de ces lieux pour la réalisation de liturgies destinées à des êtres divins différents, mais illustrant bien les capacités de fusion et fission des êtres surnaturels nahuas. Parfois, les instances dominant un espace présentent des éléments communs. Dans d'autres situations, en revanche, les êtres divins évoqués dans le rite sont la manifestation d'une union de contraires ${ }^{25}$. La reconstruction de la localisation et de l'utilisation rituelle du Totecco et du Tetamazolco ne représente qu'un exemple de l'importance de l'analyse à mener sur les pratiques rituelles réalisées dans l'aire urbaine périphérique de la capitale mexica. Enfin, les objets rituels qui jouaient un rôle majeur 
dans les cérémonies étaient les nattes de roseaux verts et blancs, les aztapilin, fabriquées pour accueillir exclusivement les différentes manifestations des entités liées à l'eau et à la terre. Une fois les rituels terminés, leur cycle de vie s'achevait dans les ayauhcalli, des lieux qui faisaient office de dépôt pour les restes des objets rituels et des offrandes, dont la charge sacrée, encore trop puissante, demandait à être occultée (Sahagún 1950-1982, II, pp. 133, 153). Là aussi, nous n'avons donné ici qu'un aperçu de l'existence de véritables liturgies végétales dont les documents coloniaux nous ont laissé des témoignages. Loin d'être un aspect secondaire du culte, l'environnement végétal des vingtaines nous renseigne sur les liens qui unissaient une divinité à une plante spécifique ; leur étude propose de nouvelles interprétations sur la dimension mythique évoquée dans le rite (Dupey García 2013) et sur la recréation de lieux mythiques dans l'aire urbaine. Ainsi, l'analyse des espaces, des mouvements et des objets végétaux utilisés aide à la tâche difficile qu'est la reconstruction de la liturgie dans son intégralité.

Lors d'etzalcualiztli, ces trois éléments évoqués illustrent la cohérence rhétorique d'un culte aux dieux aquatiques. Ils soulignent l'intégration de la vénération de l'eau, du maïs et de la terre, depuis le centre, représenté par l'enceinte sacrée, jusqu'aux sanctuaires les plus périphériques de la ville. Une pratique spatiale qui fait de Mexico-Tenochtitlan une image même du cosmos.*

* Manuscrit reçu en octobre 2013, accepté pour publication en mai 2014.

\section{Notes}

Nous remercions Anne-Sophie Gaudry et Bernadette Pernet pour les corrections orthographiques du manuscrit.

1. Dans cet article nous avons choisi de privilégier l'orthographe du nahuatl des sources historiques. S'agissant d'une langue étrangère, les formes du singulier et des pluriels seront identiques. Nous sommes consciente du fait que cette façon d'écrire ne correspond pas à la langue nahuatl. Notre choix est dicté par le souci de privilégier la clarté du contenu de notre travail. La graphie que nous présentons est celle employée par Wimmer (2006), mais nous ne marquons pas la présence des saltillos et des voyelles longues.

2. Arnold $(2009$, pp. 122, 167) identifie les localités citées comme étant géographiquement proches de San Juan Zitlaltepec.

3. Il s'agit d'une sangle de portage frontal (Wimmer 2006, s.v. mecapalli).

4. Plusieurs « vols autorisés » étaient permis aux ministres du culte pendant ce mois. Selon Botta (2004, pp. 150-151), la violence des prêtres soulignait que la gestion de la fête leur était complètement réservée. Même le pouvoir du souverain, dont le tribut pouvait à son tour être volé, leur était soumis, puisqu'ils fabriquaient des « instruments royaux », les nattes, symbole du pouvoir en Mésoamérique, afin de mener à bien leur tâche. En revanche, selon Arnold (2009, pp. 121-123) cela était dû à la crainte que les prêtres suscitaient chez les gens, pendant une vingtaine au cours de laquelle on célébrait aussi la nature violente de la consommation de nourriture par les hommes. Finalement, López Austin et López Luján (2006, pp. 40-41) considèrent que ces vols évoquaient les prouesses des dieux de la pluie dans le mythe de la découverte du maïs, une aventure mythique qui, selon les deux chercheurs, était réactualisée précisément durant etzalcualiztli.

5. Nous renvoyons, entre autres, à Caso (1967), López Austin (1970), Kirchhoff (1971). Parmi toutes ces études ressort, en raison de son caractère exhaustif, l'analyse de Graulich (1999). Entre les 
études qui ont porté sur une vingtaine particulière, on citera notamment Carrasco (1991), Olivier (1997), Limón Olvera (2001), Rodríguez Figueroa (2010) et González González (2011).

6. En dépit de l'exhaustivité du travail de Rodríguez Figueroa (2010), il importe de souligner que cet auteur fonde toutes ses interprétations exclusivement sur la production littéraire de Sahagún. Or cette approche s'avère réductrice, dans la mesure où elle exclut les informations de première main contenues dans des sources comme la Historia de las Indias (Durán 1984) et la Crónica mexicana (Alvarado Tezozómoc 2001).

7. Molina (2008, pl. 101) traduit les mots teopan et teopantli par « iglesia »; selon Siméon (1963, p. 437) teopan serait une « apocope de teopantli, temple». Finalement, Wimmer (2006, s.v. teopan; teopantli) traduit ce vocable comme un locatif dont le sens serait « près du temple, au temple ».

8. Dans le Codex de Florence le mot iteopan est employé pour désigner le sanctuaire du dieu au sommet de la pyramide, où étaient gardées les effigies divines (Sahagún 1950-1982, I, p. 35 ; III, p. 13 ; IV, p. 17), les sanctuaires qui constituaient les temples des quartiers (ibid., II, pp. 56, 99), les bâtiments rattachés au sanctuaire, tels que les lieux où l'on gardait les représentations divines ou le lieu de fabrication des effigies en pâte d'amarante (ibid., II, pp. 87, 119) ou encore en tant que synonyme de teocalli (ibid., VIII, p. 17).

9. Le Codex de Florence fait état de la réalisation de plusieurs effigies en pâte d'amarante (tzoalli), dont les lieux de fabrication étaient les sanctuaires calpulco. C'est notamment le cas des dieux Omacatl, Xiuhtecuhtli et Tzapotlantenan (ibid., I, p. 17 ; II, pp. 38, 168).

10. Les calmecac sont les suivants : Tlillan Calmecac; Mexico Calmecac; Huitznahuac Calmecac ; Tetlanman Calmecac ; Tlamatzinco Calmecac ; Yopico Calmecac ; Tzonmolco Calmecac (ibid., II, pp. 182-188).

11. Ce quartier était délimité au nord par la rue Canal del Norte, à l'est par l'Avenida del Trabajo, au sud par la croix formée par les rues Tolteca et Matamoros, jusqu'au croisement des rues Del Peñón et Jesús Carranza et, à l’ouest, par cette même rue (Caso 1956, p. 36 ; González González 2011 p. 123).

12. Une ayauhcalli était localisée à l'est : appelée Poyauhtlan, c'était le théâtre de la mise à mort, en atlcahualo, de la représentation divine appelée Poyauhtecatl ; une autre, à l'ouest, était qualifiée de Tozocan, « lieu où l'on veille » : c'est à cet endroit que les représentants des montagnes passaient la dernière nuit avant le sacrifice (Sahagún ibid., II, pp. 43, 105-106).

13. Les relations entre le dieu à peau d'écorché et la céréale sont nombreuses. Présent dans d'autres vingtaines liées au cycle du maïs, comme huey tecuilhuitl, Xipe Totec était comparé à l'épi de maïs mûr. Par ailleurs, le tlahuahuanaliztli, le «sacrifice gladiateur », du mois et de la fête tlacaxipehualiztli a été interprété comme une métaphore de la récolte des dieux - où les captifs jouaient le rôle des épis de maïs - qui se déroulait parallèlement à la celle des hommes dans les champs (Graulich 1999, pp. 299-305).

14. On ne citera ici que deux exemples : plusieurs stèles d'Izapa (400-100 av. J.-C) montrent une divinité au grand nez associée à la fois à la pluie et au maïs. Le Palais de Zacuala, à Teotihuacan, pour sa part, contient une peinture murale où un personnage, portant les « lunettes » de Tlaloc, charge sur son dos un filet rempli d'épis de maïs et tient une plante du précieux aliment dans la main droite.

15. Dans ce rite, au coucher du soleil les représentations divines des dieux réalisaient une procession (tlayahualoliztli) autour d'un édifice religieux ou de la pierre sacrificielle; elles offraient de l'encens et se dispersaient ensuite pour aller réaliser la veillée nocturne précédant leur sacrifice. La plupart des spécialistes considèrent que son étymologie et sa signification renverraient à l'entrée des victimes dans le Tlalocan (Dibble 1980 ; Graulich 1999 ; Rodríguez Figueroa 2010).

16. Cette assertion s'appuie sur le fait que, pendant le laps de temps entre les deux passages au zénith (printemps et automne), le soleil voyage vers le nord, un déplacement qui est compris entre le début et l'apogée de la saison des pluies.

17. La définition de Molina (2008, pl. 148) est « junco gordo y largo ». Siméon (1963, p. 647) traduit, lui, par " gros jonc servant à faire des nattes ».

18. Wimmer (2006, s.v. atolin) traduit par : « variété de roseau ». Puisque le mot tollin ou tullin fait référence au roseau ou au jonc le plus commun, atolin pourrait se traduire par « roseau d'eau ». 
19. Ce sceptre est arboré par les entités liées à l'eau, au maïs et au pulque, ainsi que par les prêtres des Tlaloque. Nous renvoyons, par exemple, à Sahagún (1997, pl. 261v, 263v, 264v, 265v) et au Codex Borbonicus (1991, pl. 15, 25, 29, 36). Voir aussi Vauzelle (2012, pp. 49-50).

20. Ces légumes poussaient en abondance dans le Tlalocan (Sahagún 1950-1982, III, p. 47). Le maiis, la tomate et le piment représentent aussi le don offert aux émissaires de Motecuhzoma II par Huemac, seigneur du Cincalco, autre réplique du Tlalocan (Durán 1984, II, pp. 493-497; Alvarado Tezozómoc 2001, pp. 456-457 ; Graulich 1994, pp. 311-319; Ragot 2000, pp. 140-146).

21. La même logique est à l'œuvre en ce qui concerne le langage floral (Dupey García 2013).

22. La plante appelée cochiztzapotl était employée pour soigner les troubles oculaires et dermiques, maux considérés comme envoyés par le dieu à peau d'écorché (Sahagún 1950-1982, I, p. 39 ; González González 2011, p. 274).

23. Le tolpatlactli était un roseau sauvage dont les racines, acaxilotl, pouvaient être mangées crues ou cuites dans une marmite (Sahagún ibid., XI, p. $195 ; 1989$, II, p. 783). Son étymologie souligne la relation qui existe entre ce végétal et le culte du maïs : acatl signifie roseau, tandis que le xilotl est l'épi de maïs tendre (Wimmer 2006, s.v. acatl; xilotl).

24. Les traits de l'astre couchant sous lesquels est représenté Tezcatlipoca dans son temple - dont les restes sont visibles à l'emplacement de l'édifice de l'ancien Arzobispado - semblent confirmer cette manifestation du Seigneur au Miroir fumant en tant que dieu du pulque.

25. Le Temple de Huitznahuac en huey tecuilhuitl, le Temple Xochicalco en ochpaniztli, le Temple de Coatlan en quecholli et le Temple de Xiuhtecuhtli en izcalli représentent les exemples les plus significatifs de cette union des contraires rencontrés tout au long de l'analyse des vingtaines que nous avons développée ailleurs (Mazzetto 2012).

\section{RÉFÉRENCES CITÉES}

Alvarado Tezozómoc Hernando

2001 Crónica mexicana, Gonzalo Díaz Migoyo et Germán Vázquez Chamorro (éd.), Dastin, Madrid.

ARNOLD Philip P.

2009 L'occupazione del paesaggio. Aztechied Europei nella Valle del Messico, Jaca Book, Milan.

Aveni Anthony F.

1991 "Mapping the ritual landscape : debt payment to Tlaloc during the month of Atlcahualo », in Davíd Carrasco (éd.), To change place : Aztec ceremonial landscape, University Press of Colorado, Niwot, pp. 58-73.

Barrera Rodríguez Raúl et Gabino López Arenas

2008 "Hallazgos del recinto ceremonial de Tenochtitlan », Arqueología Mexicana, 93, pp. 18-35.

BotTa Sergio

2004 Le acque preziose. Saggio sui sistemi religiosi mesoamericani, Bulzoni, Rome.

BRODA Johanna

1971 «Las fiestas aztecas de los dioses de la lluvia : una reconstrucción según las fuentes del siglo XVI ", Revista española de antropología americana, 6, pp. 245-327. 
1991a «The sacred landscape of Aztec calendar festivals : myth, nature, and society », in Davíd Carrasco (éd.), To change place: Aztec ceremonial landscapes, University Press of Colorado, Niwot, pp. 74-120.

1991b «Cosmovisión y observación de la naturaleza : el ejemplo del culto de los cerros en Mesoamérica », in Johanna Broda, Stanislaw Iwaniszewski et Lucrecia Maupomé (éd.), Arqueoastronomía y etnoastronomía en Mesoamérica, Universidad Nacional Autónoma de México, Instituto de Investigaciones Históricas, Mexico, pp. 461-500.

1997 «Lenguaje visual del paisaje ritual en la Cuenca de México », in Salvador Rueda Smithers, Constanza Vega Sosa et Rodrigo Martínez Baracs (éd.), Códices y documentos sobre México, segundo simposio, vol. II, Instituto Nacional de Antropología e Historia /Consejo Nacional para la Cultura y las Artes, Mexico, pp. 129-162.

2001 "Ritos mexicas en los cerros de la Cuenca : los sacrificios de niños », in Johanna Broda, Stanislaw Iwaniszewski et Arturo Montero (éd.), La montaña en el paisaje ritual, INAH/Conaculta, Mexico, pp. 295-317.

CALNEK Edward

1988 «The Calmecac and the Telpochcalli in Pre-Conquest Tenochtitlan », in Jorge Klor de Alva, Henry B. Nicholson et Eloise Quiñones Keber (éd.), The work of Bernardino de Sahagún, pioneer ethnographer of sixteenthcentury Aztec Mexico, Institute for Mesoamerican Studies, University of New York at Albany, coll. « Studies on culture and society » 4, Albany, NY, pp. 169-177.

CARrasco Davíd

1991 «The sacrifice of Tezcatlipoca : to change place », in Davíd Carrasco (éd.), To change place: Aztec ceremonial landscapes, University Press of Colorado, Niwot, pp. 31-57.

Caso Alfonso

1956 Los barrios antiguos de Tenochtitlan y Tlatelolco, Academia mexicana de la Historia, Mexico.

1967 Los calendarios prehispánicos, UNAM, Instituto de Investigaciones Históricas, Mexico.

Codex Borbonicus

1991 Ferdinand Anders, Maarten Jansen et Luis Reyes García (éd.), Akademische Druck- u. Verlagsanstalt/Fondo de Cultura Económica, Graz/ Mexico.

Codex de Florence $\quad$ Voir Sahagún Fray Bernardino de (1950-1982)

Codex Ixtlilxochitl

1976 Bibliothèque Nationale, Paris/Akademische Druck- u. Verlagsanstalt, Graz.

\section{Codex Magliabechiano}

1970 CL. XIII 3 (B.R. 232), Akademische Druck- u. Verlagsanstalt, Graz. 
Couvreur Aurélie

2000 Le Grand Temple de Mexico selon Fray Bernardino de Sahagún, mémoire de DEA, 2 vol., École pratique des hautes études, Paris.

Dehouve Danièle

2012 «Asientos para los dioses en el México de ayer y hoy », Estudios de Cultura Náhuatl, 44, pp. 41-64.

DibBLE Charles E.

1980 «The Xalaquia ceremony », Estudios de Cultura Náhuatl, 14, pp. 197-202.

DuPEY García Élodie

2013 «De pieles hediondas y perfumes florales. La reactualización del mito de creación de las flores en las fiestas de las veintenas de los antiguos nahuas », Estudios de Cultura Náhuatl, 45, pp. 7-36.

DURÁN Diego

1984 Historia de las Indias de Nueva España e Islas de la Tierra Firme, 2 vol., Editorial Porrúa, Mexico.

GonzÁlez Aparicio Luis

1973 Plano reconstructivo de la región de Tenochtitlan, INAH, Mexico.

GonzÁlez GonzÁLez Carlos Javier

2011 Xipe Tótec. Guerra y regeneración del maíz en la religión mexica, INAH/Fondo de Cultura Económica, Mexico.

Graulich Michel

1994 Montezuma ou l'apogée et la chute de l'empire aztèque, Fayard, Paris.

1999 Ritos aztecas. Las fiestas de las veintenas, Instituto Nacional Indigenista, Mexico.

2000 Mythes et rituels du Mexique ancien préhispanique, Académie royale de Belgique, Louvain-la-Neuve.

2005 Le sacrifice humain chez les Aztèques, Fayard, Paris.

KIRCHHOFF Paul

1971 «Las 18 fiestas anuales en Mesoamérica : 6 fiestas sencillas y 6 fiestas dobles ", in Verhandlungen des XXXVIII Internationalen AmerikanistenKongresses, Stuttgart-München. 12 bis. 18 August 1986, III, Kommissionverlag Klans Renner, Munich, pp. 207-221.

León-Portilla Miguel

1987 Los antiguos mexicanos a través de sus crónicas y cantares, Fondo de Cultura Económica, Mexico.

Leyenda de los Soles

2011 in Mitos e historias de los antiguos nahuas, Rafael Tena (éd.), Dirección General de Publicaciones del Conaculta, Mexico, pp. 174-206.

Limón Olvera Silvia

2001 El fuego sagrado : ritualidad y simbolismo entre los nahuas según las fuentes documentales, INAH/UNAM, Centro Coordinador y Difusor de Estudios Latinoamericanos, Mexico. 
LOMBARDo DE Ruíz Sonia

1973 Desarrollo urbano de México-Tenochtitlan según las fuentes históricas, INAH, Departamento de Investigaciones Históricas, Mexico.

LóPEZ Austin Alfredo

1965 «El Templo Mayor de México-Tenochtitlan según los informantes indígenas », Estudios de Cultura Náhuatl, 5, pp. 75-102.

1970 "Religión y magia en el ciclo de las fiestas aztecas », in Religión, mitología y magia, vol. II, Museo Nacional de Antropología, Mexico, pp. 3-29.

1983 «Nota sobre la fusión y la fisión de los dioses en el panteón mexica », Anales de Antropología, 20 (2), pp. 75-87.

1985 La educación de los antiguos nahuas, Secretaría de Educación Pública, El Caballito, Mexico.

1997 Les paradis de brume. Mythes et pensée religieuse des anciens Mexicains, Maisonneuve et Larose, Paris.

LóPEZ Austin Alfredo et Leonardo LóPEZ LuJÁN

2006 «Il Grande Tempio di Tenochtitlan, il Tonacatepetl e il mito del furto del mais ", in Alessandro Lupo, Leonardo López Luján et Luisa Migliorati (éd.), Gli Aztechi tra passato e presente. Grandezza e vitalità di una civiltà messicana, Carocci, Rome, pp. 23-50.

2009 Monte Sagrado-Templo Mayor. El cerro y la pirámide en la tradición religiosa mesoamericana, INAH/UNAM, Instituto de Investigaciones Antropológicas, Mexico.

MARQuina Ignacio

1960 El Templo Mayor de México, INAH, Mexico.

Mazzetto Elena

2012 Les typologies des sanctuaires mexicas et leur localisation dans l'espace sacré du Mexique préhispanique. Lieux de culte et parcours cérémoniels dans les fêtes des vingtaines à Mexico-Tenochtitlan, thèse de doctorat, Università Ca'Foscari di Venezia/Université Paris I Panthéon-Sorbonne, Venise/Paris.

Molina Alonso (de)

2008 Vocabulario en lengua castellana y mexicana y mexicana y castellana, Editorial Porrúa, Mexico.

OLIVIER Guilhem

1997 Moqueries et métamorphoses d'un dieu aztèque. Tezcatlipoca, le Seigneur au miroir fumant, Institut d'ethnologie, Paris.

Ortiz de Montellano Bernardo

1980 «Las hierbas de Tláloc », Estudios de Cultura Náhuatl, 14, pp. 287-314.

RaGoT Nathalie

2000 Les au-delàs aztèques, Archaeopress, coll. "British Archaeological Reports, International serie 881/Paris monographs in American archaeo$\log y » 7$, Oxford. 
Rodríguez Figueroa Andrea B.

$2010 \quad$ Paisaje e imaginario colectivo del altiplano central mesoamericano : el paisaje ritual en Ātl Cāhualo o Cuahuitl Ëhua según las fuentes sahaguntinas, mémoire de maîtrise, UNAM, Mexico.

Rovira Morgado Rossend

2010 «Huitznáhuac : ritual político y administración segmentaria en el centro de la parcialidad de Teopan (México-Tenochtitlan)», Estudios de Cultura Náhuatl, 41, pp. 41-64.

SAHAGÚN Fray Bernardino de

1950-1982 Florentine Codex, General history of the things of New Spain, éd. et trad. Charles E. Dibble et Arthur J. O. Anderson, 13 vol., The School of American Research, University of Utah, Santa Fe, Nouveau-Mexique.

1989 Historia general de las cosas de Nueva España, 2 vol., Alfredo López Austin et José García Quintana (éd.), Dirección General de Publicaciones del Conaculta, Mexico.

1997 Primeros Memoriales, éd. et trad. Thelma D. Sullivan, révision Henry B. Nicholson, Arthur J. O. Anderson, Charles E. Dibble, Eloise Quiñones Keber et Wayne Ruwet, University of Oklahoma Press, Norman.

SÁnchez URIARTe María del Carmen

2010 "El hospital de San Lázaro de la ciudad de México y los leprosos novohispanos durante la segunda mitad del siglo XVIII », Estudios de Historia Novohispana, 42 , pp. 81-113.

SIMÉON Rémi

1963 Dictionnaire de la langue nahuatl ou mexicaine, Akademische Druck- u. Verlagsanstalt, Graz.

SMITH Jonathan Z.

1992 To take place. Toward theory in ritual, The University of Chicago Press, Chicago.

TORQUEMADA Juan (de)

1975-1983 Monarquía Indiana, 8 vol., UNAM, Instituto de Investigaciones Históricas, Mexico.

VAUZELLE Loïc

2012 Le rapport des Aztèques à la nature : étude de l'utilisation symbolique d'éléments naturels dans les parures des divinités aztèques, d'après des textes en nahuatl du $x{ }^{e}$ siècle, mémoire de master, École pratique des hautes études, Paris.

WiMMER Alexis 